\title{
Functionalization of orange waste with phosphoric group for Anion separation
}

\author{
Kedar Nath Ghimire* \\ Central Department of Chemistry, Tribhuvan University, Kirtipur, Kathmandu \\ Email: knghimire@yahoo.com
}

\begin{abstract}
A novel adsorbent, Phosphorylated Orange Juice Residue, POJR, has been investigated. The extent of phosphorylation was measured with the aid of fluorescence $X$-ray crystallography, which indicates that the degree of phosphorylation is 1.2. The POJR has very high binding affinity for ferric ion. Also bound ferric ions selectively adsorb anions like arsenite, arsenate, selenite, selenate, antimonate and phosphate.
\end{abstract}

Keywords: Orange waste, adsorbent, oxyanions, fluorescence $X$-ray crystallography

\section{Introduction}

In the field of solvent extraction, it has been a trend to use adsorbent agents with phosphonic group like 2-ethylhexylphosphoric acids for the extraction of various metal ions. The main advantage of these materials is their high selectivity for the desired metal ions ${ }^{1}$. On the other hand, recovery and recycling of biomass wastes have become one of the promising fields of investigation in order to obtain bio-adsorbent with high affinity for metal ions ${ }^{2}$. In such circumstances, we have recently investigated another biopolymer based on orange juice residue, OJR ${ }^{3-6}$.

\section{Experimental}

Crude OJR was obtained from local juice vending centres.. It was dried in a convection oven and grounded into smaller particles with the aid of ball-mill. The powdered OJR was washed with propanol, cross-linked with epichlorohydrin and finally converted into phosphorylated form in the presence of urea using phosphoric acid. Batch wise test was carried out by taking $25 \mathrm{mg}$ of gel and $15 \mathrm{ml}$ of the corresponding metal aqueous solution in a conical flask, which was equilibrated at $30^{\circ} \mathrm{C}$. The metal ion concentrations before and after adsorption were measured by using Shimadzu Model ICPS-5000 ICP/AES Spectrometer or Shimadzu AA-6650 atomic absorption spectrophotometer, while the initial and residual concentrations of phosphate were measured by ion chromatography. All the solutions were prepared in distilled deionized water and $\mathrm{pH}$ was adjusted by hydrochloric acid or ammonia.

\section{Results and Discussion}

In order to adsorb oxyanions of arsenic, selenium, phosphorus and anions of antimony, adsorption of ferric ion on POJR was carried out in advance. It has been indicated that iron(III) was quantitatively adsorbed from the feed solution at an initial concentration of $55.8 \mathrm{ppm}$ iron over the $\mathrm{pH}$ region of 1 to 3 .

\footnotetext{
* Corresponding author
} 
The maximum uptake capacity was as high as $3.7 \mathrm{~mol} / \mathrm{kg}$. In the subsequent tests, iron(III) was loaded on POJR gel at its optimum $\mathrm{pH}$ of 3 . The high iron(III) uptake is due to the creation of better chelating environment caused by phosphorylation. The extent of phosphorylation was $1.2 \mathrm{~mol}$ of phosphorus per glucose unit of POJR ${ }^{4}$. The adsorption of iron(III) takes place by releasing protons from phosphorylated cellulose part of POJR according to cation exchange and the binding is supposed to be due to octahedral complexation, in which hydroxyl ions are exchanged by oxyanions of arsenic, selenium, antimony and phosphorus, respectively, which may be termed as ligand exchange ${ }^{3-4}$. Among them, arsenic(V), selenium(IV) and phosphorus(V) were adsorbed in acidic $\mathrm{pH}$ region; while arsenic(III) was adsorbed in alkaline $\mathrm{pH}$ region. On the contrary, antimony(III) was adsorbed in a broad $\mathrm{pH}$ range of 2-11, showing

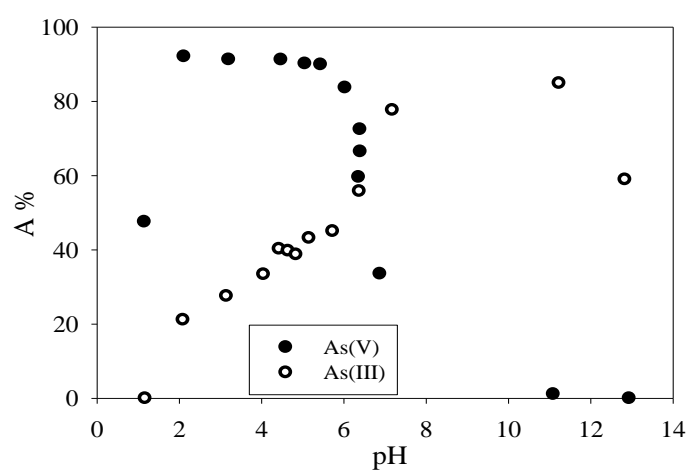

Fig.1 Adsorption of As(III, V) on Fe(III)-loaded POJR.

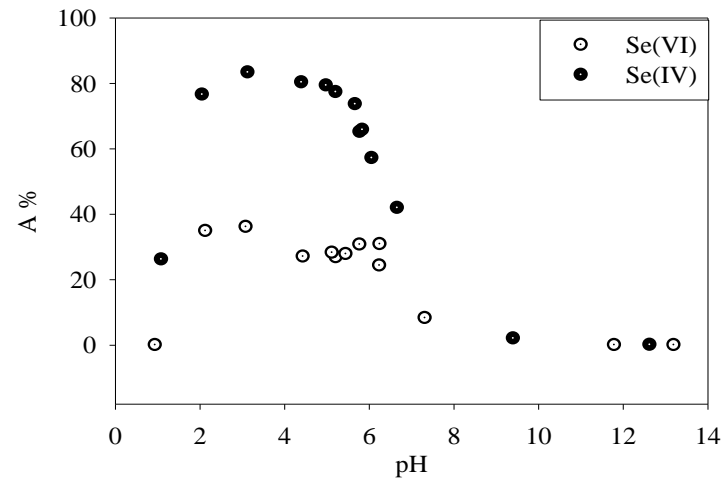

Fig.2 Adsorption of Se(IV, VI) on Fe(III)-loaded POJR.

high removal capacity even at neutral $\mathrm{pH}$ condition. Adsorption of selenium(VI) was not significant, though it has been adsorbed to some extent at acidic condition.

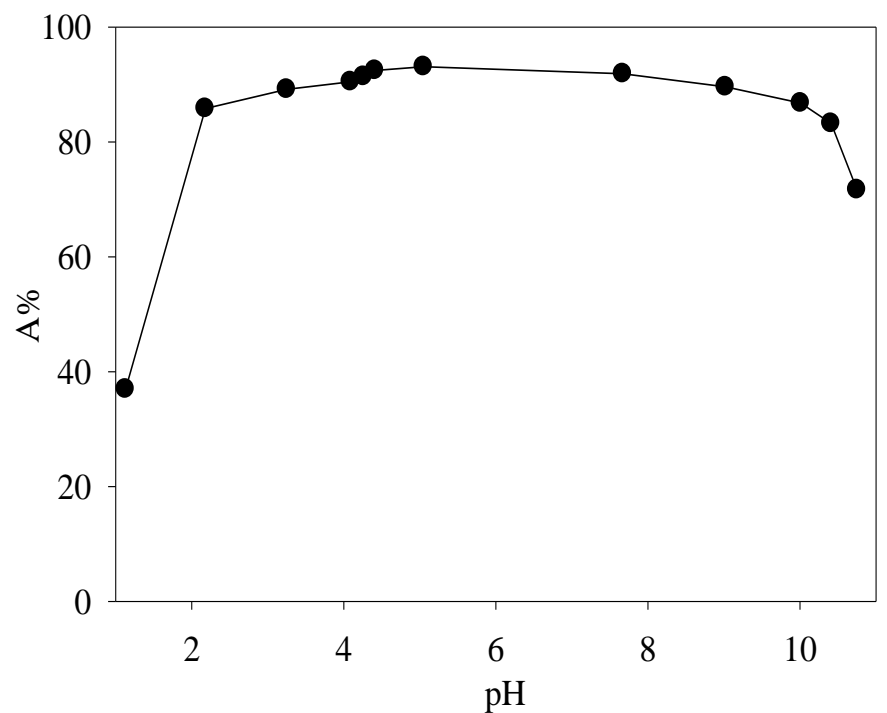

Fig.3 Adsorption of $\mathrm{Sb}(\mathrm{III})$ on Fe(III)-loaded POJR. 


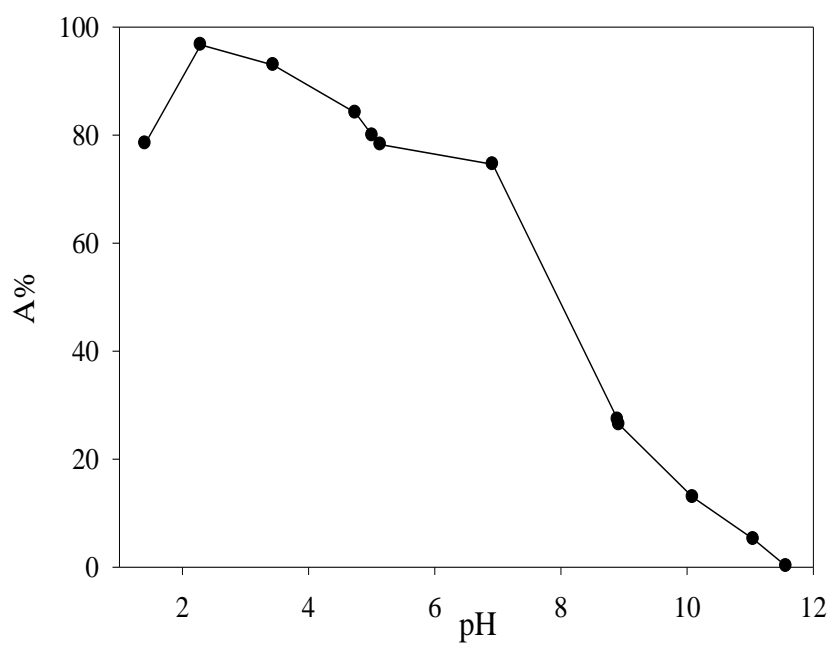

Fig.4 Adsorption of phosphorus(V) on Fe(III)-loaded POJR.

\section{References}

1. Smelov, V. S., Kondratev, V. A., Chubukov, V. V., Radiokhimiya, 1982, 24(4), 443.

2. Ghimire, K., N., Inoue, K., Miyajima, T., Yoshijuka, K., and Shoji, T., Chitin and Chitosan Research, 2001, 7(2), 61.

3. Ghimire, K. N., Inoue, K., Yamaguchi, H., Makino, K., and Miyajima, T., Water Research, 2003, 37, 4945 .

4. Ghimire, K. N., Inoue, K., Makino, K., and Miyajima, T., Sep. Sci. Technol., 2002, 37(12), 2785.

5. Inoue, K., Zhu, Y., Ghimire, K., Yano, M., Makino, K., and Miyajima, T., Proceeding of "The 6th International Symposium on East Asian Resources Recycling Technology”, pp. 264-269,October 23-25, Gyeongju, Korea, 2001.

6. Ghimire, K. N., Inoue, K., Makino, K., and Miyajima, T., Abstracts of the 9th International Conference SIS 01, Bratislava, Slovakia, 44-45, June 5-10, 2001. 